\title{
Elucidating Structure Property of Polyethylene/CNT Nanocomposite by in situ SFM Deformation Tests
}

\author{
Rameshwar Adhikari $^{1^{*}}$, Reinhold Godehardt ${ }^{2}$,Werner Lebek ${ }^{2}$, Goerg \\ H. Michler ${ }^{2}$ and Petra Pötschke ${ }^{3}$ \\ ${ }^{1}$ Central Department of Chemistry, Tribhuvan University, Kathmandu, Nepal ; ${ }^{2}$ Institute of Physics, \\ University of Halle-Wittenberg, D-06099 Halle (Saale), Germany; ${ }^{3}$ Institute of Polymer Research \\ Dresden, Hohe Str. 6, D-01069 Dresden, Germany \\ e-mail: nepalpolymer@yahoo.com
}

\begin{abstract}
Deformation behavior of nanocomposites based on an ethylene/1-octene copolymer and multiwalled carbon nanotube (CNT) was investigated by means of an atomic force microscope (AFM). Via a special tensile module integrated to an optical microscope, it was possible to record the stress-strain diagrams of the composites using miniaturized tensile specimens. By analyzing strain induced structural changes occurring at different successively applied loads, it was possible to correlate the deformation mechanisms occurring on various length scales (i.e. at macroscopic, microscopic as well as nanoscopic levels) to different degrees of deformation. It was noteworthy that, contrasting the properties of other nanocomposites described so far in the literature, both the strength and toughness of the composites were found to enhance. It was found that the deformation of the composite on nanoscopic scale was inhomogeneous owing to anisotropic properties of the CNTs and their alignments. After unloading the sample, the nanostructure of the original material was fully regenerated explaining the macroscopically observed elastomeric properties.
\end{abstract}

Keywords: Ethylene/1-octene copolymer, CNT, deformation mechanism, AFM, in situ deformation tests

\section{Introduction}

An important facet of modern materials science and engineering is to develop nanocomposite materials possessing designed property profile ${ }^{1}$. Owing to their potential to offer promising mechanical and functional properties, thus, there is a growing interest in the field of nanostructured polymeric composites. Within this context, in recent years, there has been a rapidly growing trend for the incorporation of inorganic fillers into the polymer matrix to enhance one or several physical properties. In particular, high aspect ratio fillers with platelets thickness in the range of a few nanometers (so called 'nanofiller') such as

"Corresponding author 
layered silicates have been used to increase the stiffness and strength of amorphous and semicrystalline polymers without compromising the toughness properties ${ }^{2}$. Other nanofillers include: alkaline earth metal salts, alumina, silica, polyhedral oligomeric silsesquioxanes (POSS), carbon nanotubes (CNTs) and even Fullerenes ${ }^{2-11}$. The trend in the application of nanofillers has been to 'achieve large effects with the aid of small particles'. Unfortunately, one often achieves an improvement in stiffness of the polymer at a cost of ductility. Experience shows that the deterioration of mechanical ductility of the polymeric materials by addition of nanofiller is based on the lack of affinity between the filler and the polymer, leading to the formation of agglomerates of critical sizes which may cause a premature failure of the composites. Therefore, the common practice of polymer-based nanocomposite technology has been to modify the filler surface so as to promote interaction with the polymer matrix (see ref. 12 for recent review).

Right after their invention in $1991^{13}$, the carbon nanotubes (CNTs) attracted the interest of the materials scientists as a potential candidate for nanoscale filler for different materials. Especially polymer composites containing CNTs possess an enormous innovation potential. In the polymer/CNT composites, thanks to the excellent mechanical properties (such as Moduli 200-1000 GPa, Strength 200-900 MPa), one would expect high reinforcing effects at low CNT content without loss of properties such as processability and surface gloss. On the other hand, one could also exploit the electrical conductivity of the CNTs and achieve the antistatic or conducting composite materials which have added advantages for the applications such as in electrostatic painting (such as saving of paints, environmental protection etc). As a result, in the last years such composites have been intensively investigated; and every year several thousand publications appear in scientific journals. And hundreds of conferences and symposia are devoted yearly to the CNT based composite materials.

Compared to the high expectation on the reinforced mechanical properties of the CNT filled polymer composites, the results are generally rather disappointing. The problem lies primarily on the homogenous filler dispersion in polymer matrix which should be first solved in order that the high potential of the CNT in improving the mechanical properties of polymers be achieved. Especially important in this respect is the filler-surface functionalization so that the CNT can have a good adhesion with the matrix polymer. Some of such modifications for polymers are even currently available commercially.

Equally important are the techniques by which the polymer/composite adhesion, the filler distribution and their effect on the properties of the resulting composites can be precisely evaluated. Those include different microscopic techniques which provide information about the composites' properties on different length scales. In addition to different integral methods of materials characterization, the usual microscopic techniques providing high resolution imaging of the structural details such as transmission electron microscopy (TEM), atomic force microscopy (AFM) and scanning electron microscopy (SEM) are used to characterize the structure and properties of fillers themselves as well as their polymer composites (see for example) ${ }^{14}$.

Recently, SEM was used to study the deformation behavior of epoxy/CNT composites on the basis of fracture surface analysis ${ }^{15}$. By means of AFM using aimed manipulation of the tips, it was possible not only to access the deformation behavior of the CNTs themselves 
(such as bending, kinking and stretching of the nanotubes ${ }^{16}$ ) but also to characterize their mechanical parameters ${ }^{17}$. Similar experiments were conducted using combined AFM/SEM AFM/TEM and TEM using a special stretching device ${ }^{18-21}$. In the literature, several in-situ deformation studies on the polymer/CNT composites are reported which provide a direct overview of the deformation phenomena taking place at the polymer/CNT interface, in CNT themselves and in the polymer matrix ${ }^{21-26}$. With these experiments, it was possible to draw conclusions about the reinforcing mechanisms (such as adhesion, pull out mechanism etc.).

Compared to a very rise increase in scientific publications on polymer/CNT composites, even though polyethylenes (PE) are the most important plastics from the application point of view, the number of research papers concerning the PE/CNT composites is rather limited ${ }^{27-34}$. The literature works mainly deal with percolation concentration for conducting PE/CNT composites ${ }^{27,28}$ as well as their impact on the mechanical properties of the composites ${ }^{28-34}$, also for biomedical applications and improvement of flame retarding properties for applications such as electrical cables ${ }^{29-32}$. It was demonstrated that surface modification of the CNTs plays an important role for adhesion, distribution and reinforcement effect in the polymer/CNT composites. As example, the incorporation of the virgin CNT in to the polymer could not bring any positive effect $^{28}$ whereas the composites with surface modified $\mathrm{CNT}^{32-33}$ or in situ polymerized composites $^{34}$ endowed the polymers with an improvement in mechanical performances. Moreover, the PE/CNT composites discussed in the literature deal with those with high density polyethylene (HDPE) or ultra-high molecular weight polyethylene (UHMWPE). Till this date, no systematic study has been carried out on the CNT composites based on new kinds of metallocene produced ethylene/1-octene copolymers (EOC) where the properties can be more precisely controlled over a wide range by changing the co-monomer composition as well as by blending with HDPE.

The mechanical properties of polymeric materials are determined mainly by the molecular parameters as well as the structural details (morphology) and their response to the applied load. Hence, to design polymeric materials possessing a desired property profile, it is essential to develop a suitable morphology aimed at specific micromechanical processes of deformation. Obviously, there are mainly two aspects that need to be addressed in order to exploit the potential of the CNT nanofiller and achieve a balanced reinforcement effect at minimum filler content. The first thing is to suitably design the processing route including the filler surface modification in order to achieve a optimum filler dispersion and adhesion tom the matrix. The second aspect is to control the micromechanical processes of deformation of the composites. In this regard, different microscopic techniques, especially those equipped with in-situ deformation devices, are useful to study both the morphology (filler structure, dispersion and adhesion) as well as the deformation processes at different levels (on macro, micro and nano scales).

The goal of the works lies on the elucidation of the relationship between composite morphology, mechanical properties and deformation mechanisms of the composites. For this purpose, a strategy should be developed which would lead to the knowledge of the deformation phenomena on different scales (macroscopic, microscopic and nanoscopic levels). In this communication, we show, how by developing a miniaturized tensile testing methodology, one can correlate the mechanical properties with that of deformation 
phenomena taking place in microscopic and nanoscopic levels. We present an in-situ method' by which the micromechanical properties of the composite materials can be accessed and correlated to the macroscopic true stress-strain behavior.

\section{Experimental Methods}

The matrix polymer used to prepare the composites was ethylene/1-octene copolymer (EOC) synthesized by the INSITETM technology and was supplied kindly by Dow Chemical Company. The filler was pristine commercial multiwalled carbon naotubes supplied by the company Nanocyl, Belgium.

The composites with different polymer/filler weight ratios were prepared by melt blending at $160^{\circ} \mathrm{C}$ using a twin-screw extruder followed by extrusion. Approximately 0.2 $\mathrm{mm}$ thick plaques of each sample were compression moulded at $160{ }^{\circ} \mathrm{C}$ and subsequent cooling under pressure at a rate of $10 \mathrm{~K} \mathrm{~min}^{-1}$. The specimens used in this work contain 10 wt $\%$ nanofiller.

For the AFM studies, ca. $40 \mu \mathrm{m}$ thin films sliced from the compression moulded samples by means of a Microtome were melted at a temperature of $150^{\circ} \mathrm{C}$ and allowed to cool down to room temperature at a rate of $10 \mathrm{~K} \mathrm{~min}^{-1}$ using a special miniaturised heating device. The thermal treatment was performed between two freshly cleaved mica sheets as discussed in ${ }^{15}$. Selected blends were cryo-ultramicrotomed using diamond knife and the flat surface thus formed was directly imaged by the AFM operated at tapping mode. All the AFM experiments were performed under ambient conditions. The AFM tips used were microfabricated super-sharp silicon cantilevers with a radius of curvature of about $10 \mathrm{~nm}$, and the set point ratio (ratio of the cantilever amplitude relative to its free oscillation amplitude) was approximately 0.8 .

The deformation studies were conducted under an optical microscope equipped with a miniaturized tensile testing facility. The sample surface was fixed in the strained state and the same sample area was inspected in higher magnification using atomic force microscope (D3000 Atomic Force Microscope, Digital Instruments).

\section{Results and Discussion}

\section{Morphology of ethylene/1-copolymer (EOC) and its CNT composite}

Figure 1 presents the tapping mode AFM phase images of the EOC used in this work. The sample was prepared by microtomy of a compression moulded plate followed by melting and re-crystallization.

In the phase image, owing to the high 1-octene content of this sample (35 wt.\%), in accord with the result discussed by Bensason et al. ${ }^{36,37}$, one can identify the worm-like micellar crystals of the copolymer, which resemble the crystalline fringed micelles discussed in the literature ${ }^{38}$. The motivation behind the choice of this material as a composite matrix was that it showed highly ductile behavior and that the mechanical 
properties of these kinds of copolymers could be tailored over a wide range by simply changing the comonomer content and/or by blending with high density polyethylene ${ }^{35}$.

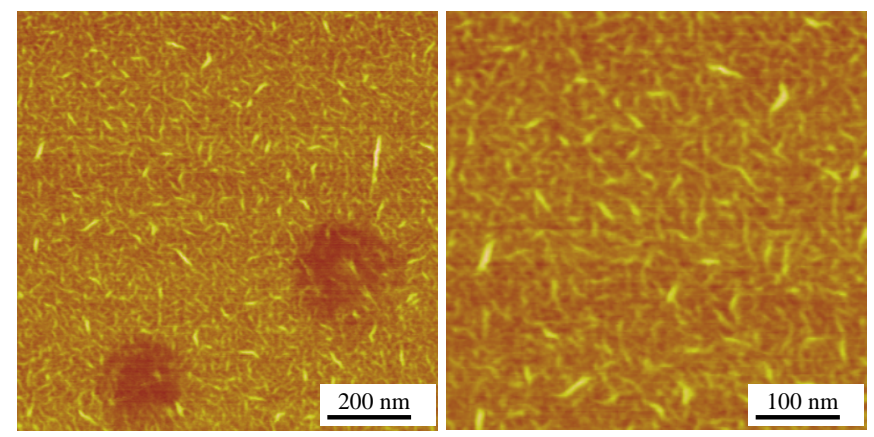

Figure 1: Different magnifications of the tapping mode AFM phase images of the EOC studied in this work

In order to gain an overview of the morphology of the composite, different microscopic techniques were employed, and the results for a composite containing $10 \mathrm{wt.} . \%$ CNT are presented in Fig. 2. The SEM result provides us an overview of the filler distribution, its adhesion with the matrix and some hints on the deformation behavior of the composites. The SEM micrograph (Fig. 2a) shows that the fracture surface is mainly covered by large flat areas, a result of reversible deformation of the polymer.

Distributed all over the surface can be visible the "white dots" which represent the small aggregates of the filler. It can be seen that the filler is well distributed into the polymer matrix. However, occasionally, large agglomerates of the filler can be found suggesting that surface modification (or functionalization) of the filler would be necessary in order to achieve better dispersion and property enhancement of the composites.

Details on the composite morphology can be found in TEM and AFM micrographs (Figs $2 \mathrm{~b}$ and c), in which even the individual CNT, frequently dispersed well in the polymer matrix can be noticed. Nevertheless, the nanotubes are not perfectly distributed as single entities rather they form small (and sometimes larger) aggregates. In the TEM micrographs, one can notice that even there are regions in which the aggregates of the impurities (shown by circles). That the nanotubes appear longer in the TEM micrographs than in the AFM image is related to the volume effect: AFM imaging is constrained on surface and subsurface regions while the TEM images also parts of CNT lying beneath the surface.

It should be mentioned that the filler morphology and resulting properties of the composites depend also much on the quality (especially the chemical purity) of the filler. Only a small amount of foreign materials, having no or too poor affinity towards the matrix would be often sufficient to give rise to large aggregates where the critical crack formation may precede.

\section{Deformation of miniaturized specimens with optical microscopic observation}

In the frame of this study, we have developed a technique to prepare special miniature tensile specimens and follow its macroscopic (which can be easily followed by optical microscopy) deformation behaviour in-situ while parallel recording the stress-strain curves. Additionally, using our stretching device, the sample can be subjected to uniaxial 
deformation up to a certain strain at a specified deformation rate under optical microscopic observation, and then it can be transferred to the AFM for investigation of the detailed structural changes occurring at nanoscopic level.
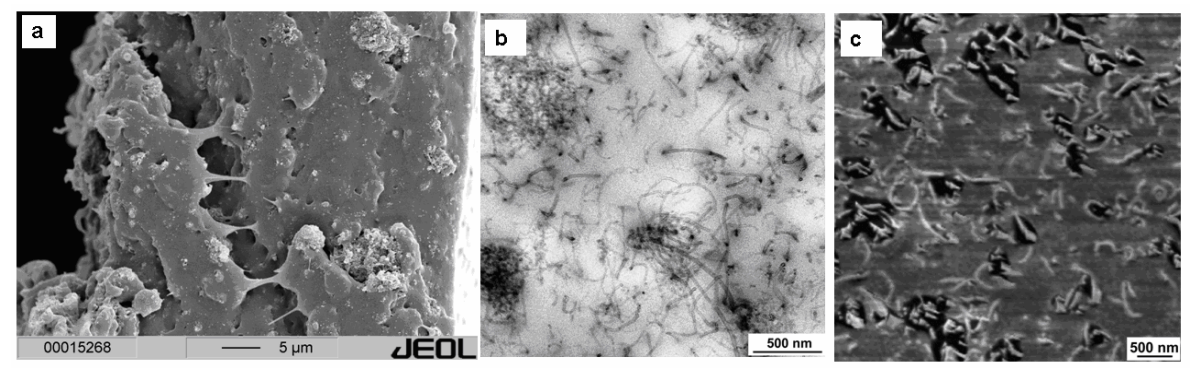

Figure 2: Morphological results via different microscopic techniques: a) SEM micrographs (overview of the morphology) of the fracture surface of the composite, b) TEM micrographs

(detailed morphology) of a ultrathin composite section and c) AFM phase micrograph

(detailed morphology) of the cryo-ultramicrotomed face of the composite

It was found that the stress-strain curves of different polymeric systems recorded using the miniaturized tensile device were surprisingly similar to those recorded by standard tensile machines using standard tensile specimens ${ }^{35}$. Figure 3 shows the stress-strain diagram of a high density polyethylene sample recorded using miniaturized specimen. The curve is very similar to that obtained using standard bulk samples. On the bottom, the optical micrographs of the tensile specimen at different stages of deformation are presented. One can see how the necking of the specimen initiates the drawing of the sample. At the initial stage of the drawing, the deformation of the sample is shear-like whereas at latter stages of the deformation, drawn region is homogeneously stretched. It should be pointed out that at any instant, the test can be interrupted, and the sample can be transferred to atomic microscope in the stretched state. Alternatively, the deformed sample can be reintegrated to the optical microscope and unloaded step-wise and again transferred to AFM for the further the study of detailed deformation mechanisms.

\section{Deformation behavior of polyethylene/CNT nanocomposite accessed by SFM}

The details about the stretching device and the results from the in-situ deformation studies, including the structural changes during both loading and unloading steps will be reported in a separate paper. In this report, we confine the discussion on the deformation of the composite during various loading steps. Figure 4 shows a series of optical micrographs (top) and AFM phase images (bottom) of the same sample locations after several deformation steps, which represent the microscopic over view and nanoscopic deformation phenomena occurring in the sample. The loading direction was from left to right in Fig. 4. A regular array of silver patter was stamped onto the surface of the tensile specimen to facilitate the finding of exactly the same specimen location after every deformation step. The AFM images provide the deformation of the composite on the nanoscale. For the sake of simplicity we may concentrate on two areas indicated by circles and squares. By inspecting the distance changes between the patterns one can estimate the deformation behavior of the composite on macroscopic scale, which appears to be homogeneous over the whole investigated range. The circle in Fig. 4b shows a single CNT which lies in a bent 
stage in the polymer matrix. The applied stress causes the bent CNT to become almost straight one (Fig. 4h) with increasing strain. With increasing deformation, several CNTs disappear from the surface simply because the scanned area is smaller than the original area due to high deformation. The square in Fig. $4 \mathrm{~b}$ contains an aggregate of carbon nanotubes, which lie seemingly perpendicular to the scanned surface. With progressing deformation the aggregate is destroyed leading to the scattered tubes. There is clearly a separation of the filler tubes which also appear longer at higher deformation (Fig. 4h), a sign of partial pull out of the nanotubes. On releasing the stress step by step, the original structure of the nanocomposite structure were fully regenerated which explains the largely reversible deformation of the composite. It was further observed that, in contrast to the CNT modified polymer composites discussed so far in the literature, a clear reinforcement effect was noticed.

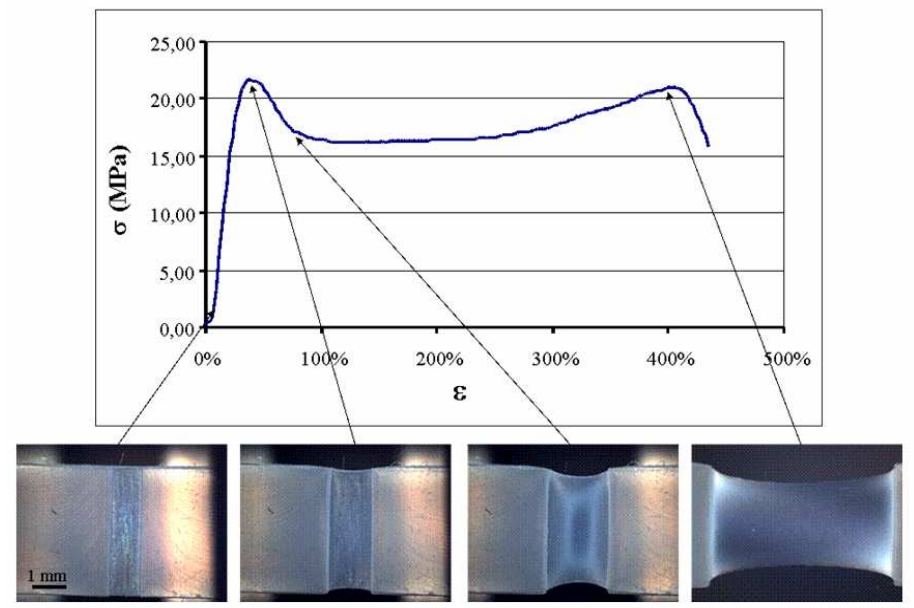

Figure 3: Tensile deformation test of an HDPE sample under optical microscopic control; the optical micrographs of the samples were recorded at different nominal strains; the macroscopic deformation structures and corresponding locations in the stress-strain diagrams are shown

\section{Conclusions}

The deformation behavior of the nanocomposite of ductile thermoplastic (the EOC) and multi-walled carbon nanotube has been studied for the first time in situ using optical microcopy and scanning force microscopy (SFM). By analyzing the strain induced structural changes occurring at successive loads applied, it was possible to correlate the deformation mechanisms occurring on various length scales to different degree of deformation. Contrasting the properties of other nanocomposites described so far in the literature, the composite strength was enhanced while maintaining their toughness. Macroscopically the pure copolymer as well as its composites deformed in a homogeneous manner. It was found that the deformation of the composite on nanoscopic scale was inhomogeneous owing to the anisotropic properties of the high aspect ratio CNTs. After the load removal, the 
nanostructure of the original material fully regenerated explaining the macroscopically observed elastomeric characteristic of the composite.

$\varepsilon=0$
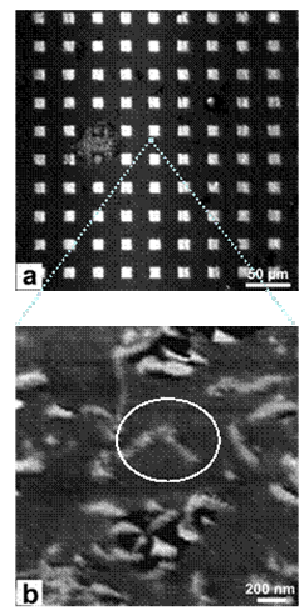

$\varepsilon=0.36$
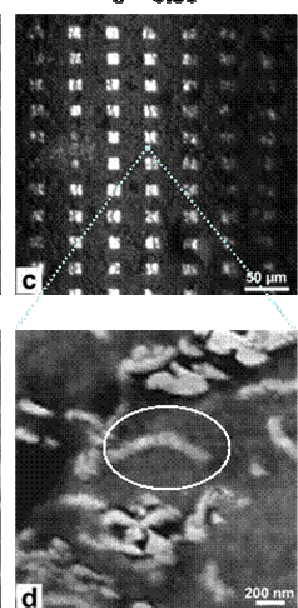

$\varepsilon=0.75$
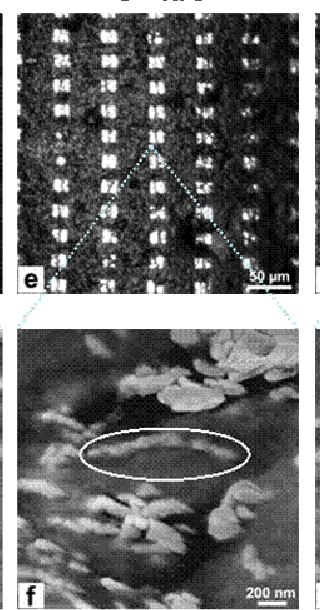

$\varepsilon=1.55$
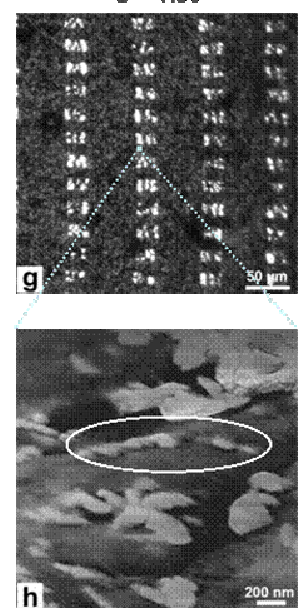

Figure 4: Optical microscopic images (top) \& tapping mode AFM phase micrographs (bottom) of EOC/CNT composite surface at different total strains indicated; micrographs were collected after every consecutively increased strain; squares and circles are meant to guide the reader's eye; deformation direction is horizontal

\section{Acknowledgements}

RA is grateful to the Alexander von Humboldt Stiftung for supporting his research stay in Germany (Feb.-July, 2009) through Georg Forster Fellowship. He is further indebted to Nepal Academy of Science and Technology (NAST) for providing Science Promotion Grant. This work was supported by State Government of Saxony-Anhalt in the frame of the project - "Cluster of Excellence:Nanostrukturierte Materialien".

\section{References}

1. B. Bhushan (Ed.), Handbook of Nanotechnology, Springer, Berlin, 2003

2. R. A. Vaia, H. Ishii and E. P. Giannelis, Chem. Mater. 2003, 5, 1694

3. F.J. Baltá Calleja, L. Giri, T. Asano and T. Yoshida, Rep. Fac. Sci. Shizuoka Univ. 1994, 28, 53

4. B. J. Ash, J. Stone, D. F. Rogers, L. S. Schadler, R. W. Siegel, B. C. Benicewicz and T. Apple, Materials Research Society Symposia Proceeding, 2001, Vol. 661

5. B. J. Ash, R. W. Siegel and L. S. Schadler, J. Polym. Sci.: B: Polym. Phys. 2004, 42, 4371

6. R. Adhikari, S. Henning and G. H. Michler, Macromol. Symp. 2006, 233, 26

7. B. X. Fu, A. Lee and T. S. Haddad, Macromolecules 2004, 37, 5211

8. H. Koerner, W. Liu, M. Alexander, P. Mirau, H. Dowty and R. A. Vaia, Polymer 2005, 46, 4405

9. P. Poetschke, A. R. Bhattacharyya and A. Janke, Polymer 2003, 44, 8061 
10. I. Szleifera and R. Yerushalmi-Rozen, Polymer 2005, 46, 7803

11. C. Wang, Z.-X. Guo, S. Fu, W. Wu and D. Zhu, Prog. Polym. Sci. 2004, 29, 1079

12. A. Usuki, N. Hasegawa and M. Kato, Adv. Polym. Sci. 2005, 179, 135

13. S. Iijima, Nature 1991, 354, 56

14. P. Pötschke, A. R. Bhattacharyya, A. Janke, Eur. Polym. J. 2004, 40, 137

15. P. M. Ajayan, L. S. Schadler, C. Giannaris and A. Rubio, Adv. Mater. 2000, 12, 750

16. M. R. Falvo, G. J. Clary, R. M. Taylor II, V. Chi, F. P. Brooks Jr, S. Washburn and R. Superfine, Nature 1997, 389, 582

17. E. W. Wong, P. E. Sheehan and C. M. Lieber, Science 1997, 277, 1971

18. M.-F. Yu, B. I. Yakobson and R. S. Ruoff , J. Phys. Chem. B 2000, 104, 8764

19. A. H. Barber, R. Andrews, L. S. Schadler and H. D. Wagner, Appl. Phys. Lett. 2005, 87, 1-3

20. C. A. Cooper, S. R. Cohen, A. H. Barber and H. D. Wagner, Appl. Phys. Lett. 2002, 81, 3873

21. O. Lourie and H. D. Wagner, Appl. Phys. Lett. 1998, 73, 3527

22. C. Bower, R. Rosen, L. Jin, J. Han and O. Zhou, Appl. Phys. Lett. 1999, 74, 3317

23. D. Qian, E. C. Dickey, R. Andrews and T. Rantell, Appl. Phys. Lett. 2000, 76, 2868

24. G. L. Hwang, Y.-T. Shieh and K. C. Hwang, Adv. Funct. Mater. 2004, 14, 487

25. D. Qian and E. C. Dickey, J. Microscop. 2001, 204, 39

26. R. Andrews, D. Jacques, D. Qian and T. Rantell , Acc. Chem. Res. 2002, 35, 1008

27.P. Pötschke, A. R. Bhattacharyya and A. Janke, Polymer 2003, 44, 8061

28. T. McNally, P. Pötschke, P. Halley, M. Murphy, D. Martin, S. E. J. Bell, G. P. Brenman, D. Bein, P. Lemoine and J. P. Quinn, Polymer 2005, 46, 8222

29. Y. Bin, M. Kitanaka, D. Zhu and M. Matsuo, Macromolecules 2003, 36, 6213

30. S. L. Ruan, P. Gao, X. G. Yang and T. X. Yu, Polymer 2003, 44, 5643

31. R. Zhang, C. Reynolds, P. Ciselli and T. Peijs, Proc. Carbon Nano Tube (CNT) Polymer Composites International Conference (Sept. 4 - 7, 2005, Hamburg), pp. 204

32. M.-R. Babaa, M. Holzinger, S. Fiorito, P. Poncharal and A. Zahab, Proc. Carbon Nano Tube (CNT) - Polymer Composites International Conference (Sept. 4 - 7, 2005, Hamburg), pp. 49

33. Y. Zou, Y. Feng, L. Wang and X. Liu, Carbon 2004, 42, 271

34. X. Tong, C. Liu, H.-M. Cheng, H. Zhao, F. Yang and X. Zhang, J. Appl. Polym. Sci. 2004, 92, 3697

35.R. Adhikari, R. Godehardt, W. Lebek, S. Frangov, G. H. Michler, H.-J. Radusch and F. J. Baltá-Calleja, Polym. Adv. Technol. 2005, 16, 156

36. S. Bensason, J. Minick, A. Moet, S. Chum, A. Hiltner and E. Baer, J. Polym. Sci.: Part B: Polym. Phys. 1996, 34, 1301

37. S. Bensason, S. Nazarenko, S. Chum, A. Hiltner and E. Baer, Polymer 1997, 38, 3513

38. L. H. Sperling, Introduction to Physical Polymer Science. $3^{\text {rd }}$ edition, Wiley Interscience, New York 2001, pp. 217 